\title{
I ntracephalic glomus tumor presenting as peripheral neuropathic pain and literature review
}

\author{
Mohammed Ahmed Saadah ${ }^{1}$, Mahfoud El Beshari ${ }^{1}$, Mwaffaq Al Heis ${ }^{2}$, Loai M Saadah ${ }^{3}$, Amani Ali \\ Al Zaabi ${ }^{1}$, Abdul Rahman Al Bloushi ${ }^{4}$, Gamal Rashid ${ }^{5}$ \\ 1. Department of Neurology, Zayed Military Hospital, Abu Dhabi, UAE. 2. Department of Radiology, Zayed Military Hospital, \\ Abu Dhabi, UAE. 3. Department of Pharmacy, Zayed Military Hospital, Abu Dhabi, UAE. 4. Department of Cardiovascular \\ Surgery, Zayed Military Hospital, Abu Dhabi, UAE. 5. Department of Pathology, Zayed Military Hospital, Abu Dhabi, UAE \\ Correspondence: Mohammed Ahmed Saadah. Address: Department of Neurology, Zayed Military hospital, Abu Dhabi, \\ UAE. Email: mohdsaadah@yahoo.com
}

Received: November 19, 2015

Accepted: December 28, 2015 Online Published: January 20, 2016

DOI : 10.5430/ijdi.v3n1p55

URL: http://dx.doi.org/10.5430/ijdi.v3n1p55

\section{Abstract}

Background: Glomus tumors are benign highly vascular neoplasms, originating from glomus body and sprout in subcutaneous layer of acral parts, particularly subangual or digital pulp locations. Extra digital glomus tumors were reported in palm, feet, ear, tongue, nose, mouth, vagina and glans penis. Extra cutaneous glomus was rarely seen in gastrointestinal tract, mesentery, mediastinum, trachea, and cervix, with infrequent occurrence in muscles, bones, periosteal, and intraneural layers. The subcutaneous layer of forearm is the most common extra digital location; however, it is exceedingly rare in intravascular layer of forearm. We are reporting an intracephalic glomus tumor presenting as peripheral neuropathic pain. This presentation was not previously reported in literature.

Case report: A 38-year-old male presented with two years history of spontaneous and nocturnal right forearm pain, provoked by exposure to cold, light touch or brushing of skin. Non painful stimulus and painless touch evoked allodynia, and hyperesthesia respectively. Neurologic examination was negative apart from focal tenderness with no sensory alterations. Grey scale and colored ultrasound, MR and CT venography demonstrated an echo poor small round and avidly enhancing lesion in right cephalic vein. Complete surgical excisional resection was performed. Gross examination revealed dark-red, well defined soft tissue tumor, and histopathology confirmed glomus tumor. Symptoms completely resolved postoperatively.

Conclusion: Intravascular glomus tumors rarely occur in forearm; and exceedingly rare to present as neuropathic pain, therefore, comprehensive history, and thorough physical examination, neuroimaging techniques, and early surgical excision will likely prevent delayed or incorrect diagnosis.

\section{Keywords}

Glomus, Glomangioma, Extra digital, Intravascular, Neuropathic pain, MRI

\section{I ntroduction}

The digital glomus represents approximately $1 \%-2.6 \%$ of all hand tumors ${ }^{[1-3]}$. They are well recognized as painful subcutaneous nodules in subungual or digital pulp locations ${ }^{[4,5]}$. They are more common in women between 30 and 50 
years of age. Extra digital cases account for $11 \%-65 \%$ of all glomus tumor ${ }^{[6]}$. Extra digital glomus tumors have been reported in the palm, wrist, forearm, elbow, feet, tympanic membrane, tongue, nose, mouth, vagina and glans penis ${ }^{[7,8]}$. They have been reported to be more common in men. Extra cutaneous glomus tumors were reported rarely in the stomach, gastric antrum, colon, mesentery, mediastinum, trachea, and cervix ${ }^{[8,9]}$. The forearm is the most common extra digital location, and the lesions most commonly occur in the subcutaneous layer. Extra cutaneous glomus tumors occur very infrequently in locations such as muscle, bone, periosteal, intravascular and intraneural layers ${ }^{[10,11]}$. They are usually solitary but a multiple glomus tumor syndrome has been described and is transmitted in an autosomal dominant pattern ${ }^{[9]}$. The etiology of glomus tumor is not clear.

The glomus body is a normal specialized arteriovenous malformation that plays a role in thermoregulation ${ }^{[10]}$. Glomus tumor is a benign highly vascular neoplasm composed of modified smooth muscle cells and is believed to originate from the glomus body within the reticular dermis. These tumors are most frequently found in the extremities, usually on the digits. The forearm is the most common extra digital site, however, the intravascular origin of a forearm glomus tumor is an exceedingly rare occurrence. To the best of our knowledge, only 3 cases of intravascular forearm glomus tumors have been reported ${ }^{[10,11]}$. We will present an interesting rare case of a patient with chronic peripheral neuropathic pain and cold intolerance caused by an intravascular glomus tumor in the forearm. The combination of this particular location and peripheral neuropathic pain was not previously reported in the World literature.

\section{Case presentation}

A 38-year-old male referred from the neurosurgical clinic as a case of unexplainable neuropathic pain. He was previously seen by the orthopedic surgeon for a vague swelling and severe pain of his right elbow and upper forearm. Routine extensive laboratory investigations, plain X-ray, ultrasound and MRI elbow and forearm were reportedly normal. The pain is severe, spontaneous and nocturnal right upper forearm pain that radiates down to the hand and to the upper arm. It is provoked by exposure to cold currents of the air condition, light touch or brushing of a localized skin area on the upper right forearm. Normally non painful stimulus resulted in severe pain that persisted for longer time after the stimulus (allodynia). In addition, normally painless touch stimulus evoked a markedly painful sensation (hyperesthesia); normally painful stimulus disclosed extreme painful reaction (hyperalgesia) and painful stimuli manifested exaggerated levels of pain (hyperpathia).

Local examination of that forearm and whole body screening, neurologic and systemic examination showed no signs, however, on deep palpation under sedation, a small pea like swelling in the high upper medial volar aspect of the right forearm was felt and this has been exquisitely tender despite $10 \mathrm{mg}$ valium given intravenously before examination. Differential diagnosis included shrapnel, peripheral or central neuropathic pain, neuroma, glomus tumor, hemangioma, epithelial inclusion cyst, and giant cell tumor of tendon sheath. Grey scale ultrasound showed an echo poor round lesion in the lumen of right cephalic vein and it is $4 \mathrm{~mm} \times 3.7 \mathrm{~mm}$ in diameter (see Figure 1A). Colored doppler revealed that the lesion is deviating the blood flow but not completely blocking the vein (see Figure 1B). CT scan without contrast did not show any metallic objects. CT and MRI using 1.5 and 3 tesla scanners without contrast were normal. Venous phase of CT angiography showed a slightly hyperdense and an avidly enhancing subcutaneous lesion in the right cephalic vein just below the level of the elbow joint (see Figure 1C). T1Fat Sat contrast enhanced MRI disclosed an avidly enhancing subcutaneous roundish lesion in the same previously mentioned site (see Figure 1D). During the operation, a $3 \mathrm{~cm}$ longitudinal incision was made on the radial aspect of the right elbow at the site of the painful vague swelling (see Figure 2A). A dark-red, well-defined, and oval-shaped mass (diameter, $4 \mathrm{~mm}$ ) was identified in the right cephalic vein (see Figure 2B). The mass was carefully resected from the vein which was then ligated. Surgical excision revealed the dark red round intravascular lesion. Gross macroscopical examination confirmed the diagnosis of glomus tumor (see Figure 2C). Histopathological examination showed large uniformly rounded cells with centrally located round nuclei and eosinophilic cytoplasm, and surrounded by obvious capillary vessels (see Figure 2D). The tumor was contiguous with the smooth muscle layer of the affected right cephalic vein. Although the tumor was clearly vascular, the vascularity was 
not exceptionally suggestive of glomangioma which is the only differential feature between the two entities which are used interchangeably in practice. Immunohistochemical staining revealed that the tumor cells were positive for $\alpha$-smooth muscle actin and vimentin. Genetic testing was negative for glomulin mutation. After the complete surgical excision, the patient's symptoms fully resolved within few days. There was no recurrence of pain up to 4 years postoperatively.

Figure 1. Arrows. A: Grey scale; B: Duplex venous ultrasound showing echo poor lesion, occluding right cephalic vein, just above the elbow; C: Venous phase of CT angiography; D: T1 Fat Sat contrast enhanced MRI

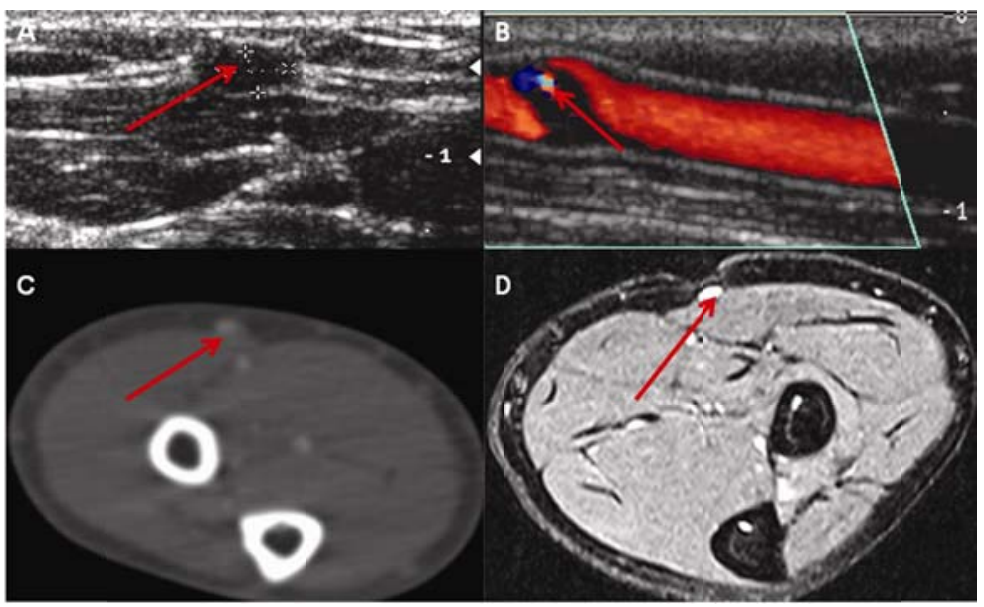

Figure 2. A. Vascular incision; B. Round pale opaque neoplasm; C. Grossly round highly vascular glomus tumor; D. Consisting of round cells and nuclei

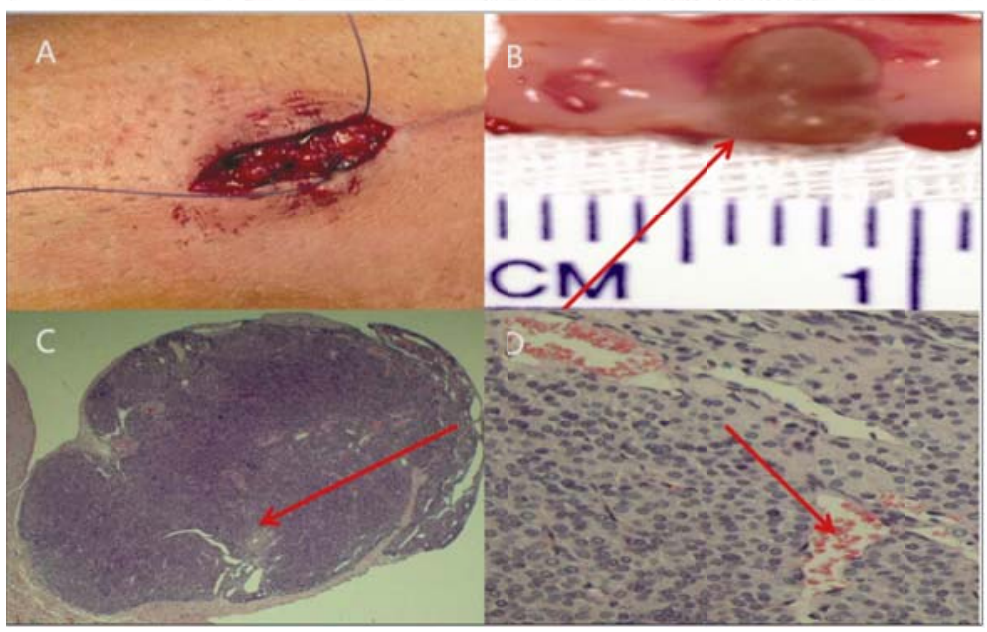

\section{Discussion}

The normal glomus body is involved in thermal regulation through control of the skin circulation. It is composed of an afferent arteriole, which is derived from the small arterioles supplying the dermis ${ }^{[8]}$. Glomus tumors are considered to be a hamartomatous proliferation of modified smooth muscle cells originating from normal glomus cell populations. Glomus tumors are soft tissue tumors of the hand and are well recognized by the dermatologist or the orthopedic surgeons as painful subcutaneous nodules in subungual or digital pulp location ${ }^{[11]}$. Importantly, extra digital cases are reported to be more common in men, although most subungual lesions occur in women ${ }^{[10,11]}$. The etiology of glomus tumor is not clear, although an autosomal dominant pattern of inheritance and history of injury have been reported. We present the case of a soldier who had no family history of a relevant glomus tumor and no history of injury; however, chronic peripheral neuropathic pain and focal tenderness were the main presenting symptoms and were completely alleviated after surgical removal of this fascinating extra digital forearm gloms tumor.

The classic triad symptoms of a glomus tumor are severe paroxysmal pain, localized point tenderness and cold intolerance. Contrary to the prevailing literature, glomus tumors frequently present with neuropathic pain, particularly when they are not associated with local skin changes or palpable masses ${ }^{[12]}$. A glomus tumor can occasionally cause pain by directly 
compressing the forearm nerves ${ }^{[9,13,14]}$. However, glomus cells are adjacent to a rich neural bed; this may explain the severe neuropathic pain and cold hypersensitivity associated with these lesions. In addition, mast cells of the glomus tumor may play an important role in the mediation of neuropathic pain ${ }^{[15,16]}$. Spontaneous pain was observed in $80 \%$ of patients ${ }^{[4]}$, sensitivity to touch in $100 \%$ and cold sensitivity in $63 \%{ }^{[4,6]}$. In one series, $100 \%$ presented with severe pain, and point tenderness while $78 \%$ had cold sensitivity ${ }^{[6]}$. Excruciating, paroxysmal pain is pathognomonic of glomus tumor. Interestingly, all these characters are genuine features of neuropathic pain. As previously mentioned, the forearm is the most common extra digital location, and the lesions most commonly occur in the subcutaneous layer, with very infrequent occurrence in extra cutaneous locations such as muscle, bone, and blood vessels ${ }^{[10,15]}$. Superficially located glomus tumors often produce typical symptoms such as paroxysmal pain, exacerbated by changes in temperature ${ }^{[9]}$. However, glomus tumors located at an unusual site may present with features of no pain or hypersensitivity ${ }^{[14,15]}$. Interestingly, those located at unusual extra cutaneous sites may or may not present with features of neuropathic pain ${ }^{[8,9,15]}$. Indeed, there was no sensory alteration in the current case in which the glomus tumor was located intravascularly. A retrospective comparative study ${ }^{[17]}$ of the clinical and pathological characteristics of extra digital and digital glomus tumors revealed that patients with extra digital tumors were significantly older $(P=.009)$, with significantly greater male predominance $(P<.001)$. Our index case is 38 years old male. It is difficult to objectively detect a glomus tumor through clinical palpation because of the subcutaneous or deeper layer location and the small size (few millimeters); however, we were able to palpate this small vague swelling under sedation with $10 \mathrm{mg}$ valium given intravenously before palpation.

This amazing neoplasm was recently classified, according to the prevailing cell type into glomus tumors, glomangiomas and glomangiomyomas with predominant glomus cells, blood vessels or smooth muscle cells respectively ${ }^{[18,19]}$. However, glomangiomas are best considered as glomuvenous malformations rather than tumors. Glomus tumors are typically solitary, $1 \mathrm{~cm}-2 \mathrm{~cm}$ reddish blue papules or nodules. They usually present as paroxysmal pain, focal tenderness and cold intolerance. Neuropathic pain results from damage or disease affecting the somatosensory system ${ }^{[20]}$. It may be associated with abnormal sensations called dysesthesias, and pain produced by normally non-painful stimuli (allodynia). Neuropathic pain may have continuous and/or episodic (paroxysmal) components. The latter are likened to an electric shock. Common qualities include burning or coldness, "pins and needles" sensations, numbness and itching. The paroxysmal electric like nature, radiating up wards or down wards along the forearm and provoked by the cold of the air condition at night and the longer duration of pain following non painful stimuli is highly suggestive of peripheral neuropathic pain (allodynia). Nociceptive pain, by contrast, is more commonly described as aching. However, combined nociceptive and neuropathic pain does occur in clinical practice as seen in the famous and popular low back pain. Up to $7 \%$ to $8 \%$ of the European population is affected and in $5 \%$ of persons it may be severe ${ }^{[21]}$. Neuropathic pain may result from disorders of the peripheral nervous system or the central nervous system (brain and spinal cord). Thus, neuropathic pain may be divided into peripheral, central, or mixed (peripheral and central) neuropathic pain.

Glomus is usually tender, or extremely painful, particularly following changes in temperature or pressure. They commonly affect the nail bed or the finger pulp. Microscopically, there are solid sheets of glomus cells around small blood vessels. Glomangiomas, are less common, and are typically found in childhood. They are usually multiple, soft, reddish blue nodules or pink to deep blue plaque like lesions. They are often widespread, and may join together to become larger plaques. They tend to get thicker and bluer with age. Although they start off painless, they may become tender to touch. Histologically they resemble venous malformations with large, dilated, thin-walled veins in the dermis and subcutaneous tissue, but have clusters of $\alpha$-actin-positive glomus cells lining the venous spaces ${ }^{[22]}$. Most of them is sporadic, however, segmental multiple lesions with an autosomal dominant pattern of inheritance, incomplete penetrance and multiple deletions of the responsible gene were reported ${ }^{[2,23]}$. An abnormal chromosome 1p21-p22 has been identified and it is associated with damage to a protein called glomulin ${ }^{[24]}$. Earlier reports have shown families with inherited glomuvenous malformations (GVMs) with linkage to chromosome 9p21, the mutation caused ligand independent activation of an endothelial cell-specific receptor tyrosine kinase ${ }^{[25]}$. These malformations are caused by the novel gene that may act to regulate angiogenesis. These multiple GVMs are rare and often congenital, but they may also appear later in adult life ${ }^{[22]}$. 
The exact incidence of glomus tumors is unknown. The multiple variant, accounts for less than $10 \%$ of all cases ${ }^{[3,5]}$. Due to misdiagnoses of many of these lesions as hemangiomas or venous malformations, an accurate assessment of the incidence is extremely difficult. The most common clinical feature is pain, which is usually associated with solitary lesions. Multiple tumors are less likely to be painful. Solitary glomus tumors are more frequent in adults, and are more common in females while multiple lesions are slightly more common in males. Multiple glomus tumors develop 10-15 years earlier than single lesions; about one third of these tumors occur in those younger than 20 years ${ }^{[5]}$. Congenital glomus tumors are rare; they are plaque like in appearance and are considered a variant of multiple glomus tumors. The peculiar Kasabach-Merritt syndrome with more than 400 glomus tumors and thrombocytopenia as a result of platelet sequestration has been reported ${ }^{[2]}$.

The diagnosis is primarily clinical. Localization of the tenderness to an area and a size of a pinhead are supportive of the diagnosis. However, several clinical tests may be useful for diagnosis. These include Love's pin and Hildreth's tests ${ }^{[17]}$. The Love's pin test is positive, when the patient experiences severe pain and the pain is reduced when the skin overlying the tumor is pressed with a pinhead, or ballpoint pen. This test was $100 \%$ sensitive and specific in one series ${ }^{[3,25]}$. The cold sensitivity test is positive when immersing the hand in cold water elicits severe pain in and around the area. History of cold weather aggravating the symptoms is additive. Hildreth's test is another reliable clinical sign in the diagnosis of these tumors ${ }^{[3]}$. The Hildreth's test and the Love's pin test are reliable methods of diagnosing glomus hand tumors with sensitivity and specificity exceeding $90 \%{ }^{[3]}$. The test is performed by elevating the patients' arm to exsanguinate it. A tourniquet is inflated to $250 \mathrm{mmHg}$ and on palpating the area, less pain and tenderness will be evoked. The test is positive when on releasing the cuff, a sudden rebound of pain and tenderness is observed. This test had a sensitivity of $92 \%$ and specificity of $91 \%$; however, half of the cases involved in the above study were not glomus tumors ${ }^{[16]}$.

Definitive diagnosis is only possible after surgical excision and evaluation of the lesion by histopathology. Only $20 \%$ of extra digital glomus tumors were diagnosed correctly by the initial physician. Misdiagnosis and delayed diagnosis are common. The average duration of symptoms was greater than 7 years, with most patients being previously misdiagnosed. Improved approach regarding diagnosis and treatment of these neoplasms may reduce morbidity, ensuing chronic pain, and psychiatric consequences of delayed diagnosis or misdiagnosis. Compared to the subungual glomus tumor, extra digital glomus tumors may show atypical features, and the absence of objective physical signs often leads to delay in diagnosis. A comprehensive approach, including careful clinical history, thorough physical examination, observation of the disease progress, and imaging studies, is essential upon clinical suspicion. Although glomus tumors are rare, the neurologist should be aware of them in the differential diagnosis of asymmetric peripheral neuropathic pain in the limbs. These tiny masses can cause severe neuropathic pain, and a long history may unfortunately result in an improper diagnosis such as neuralgia, depression, and psychosomatic pain. High index of clinical suspicion plays an important role in management, and only early surgical extirpation alleviates this terrible pain.

As a preoperative imaging for glomus tumors, scintigraphy is a useful but nonspecific diagnostic tool. Moreover, ultrasonography is capable of demonstrating the size, site, and shape of the tumor, but is frequently influenced by the surgeon's experience. MR imaging, however, is an excellent diagnostic tool for detecting the soft-tissue origin of a glomus tumor as small as $2 \mathrm{~mm}$. This method can also accurately define the location and limits of a tumor ${ }^{[3,4,26]}$. MRI in particular can verify the presence of small soft tissue tumors such as extra digital glomus tumors, epidermal cysts, fibromas, synovial cysts, and venous malformations. A review of the literature on MRI findings of extra digital glomus tumors showed that these lesions are oval-shaped and well defined and demonstrate hypo- or isointensity on T1-weighted images and hyper intensity on T2-weighted images. In the present case, T1-weighted MRI images showed an intermediate intensity, and T2-weighted images showed an inhomogeneous high signal ${ }^{[4,5,26]}$. However, in a previous study, MRI failed to reveal any lesion, and the main role of these imaging studies is to identify the presence of a lesion. A definitive diagnosis is only established in a histologic examination performed after surgical excision; this examination can identify glomus tumor cells, which are distinct uniformly rounded cells with centrally located round nuclei. 
Cutaneous and extra cutaneous glomus tumors are biologically benign. Malignant glomus tumors, or glomangiosarcomas, are extremely rare and usually represent a locally infiltrative malignancy. However, metastases are exceedingly rare, although they do occur and are usually fatal ${ }^{[6,8]}$. Nonetheless, malignant variant may appear de novo. It tends to appear as a painful nodule located at the same sites. It is considered a low grade malignant neoplasm with tendency for local recurrence ${ }^{[6]}$. However, wide spread metastases of a malignant glomus tumor involving the skin, lungs, jejunum, liver, spleen, and lymph nodes have been reported ${ }^{[27,28]}$. Criteria for the diagnosis of malignancy include tumor size of more than 2 centimeters, subfascial or visceral location, atypical mitotic figures, marked nuclear atypia and any level of mitotic activity ${ }^{[27,29]}$. However, recent classification of malignant glomus tumors into three categories based on their histologic evaluation is as follows: locally infiltrative glomus tumors (LIGT), glomangiosarcomas arising in benign glomus tumors (GABG), and glomangiosarcomas arising de novo (GADN) ${ }^{[29]}$. These malignant tumors have been reported usually in older age groups, and at several locations, primarily the soft tissues and gastrointestinal tracts. Our case showed no malignant features such as marked cytological atypia, increased mitotic activity, or infiltrative growth pattern. Treatment consists of complete local excision and close surveillance ${ }^{[24]}$.

As no medical treatment exists, surgical extirpation was the preferred treatment and provided immediate and sustained pain relief. Extra digital tumors were successfully treated with simple excision without recurrence in $89.5 \%{ }^{[17]}$. Immunohistochemical studies of the excised specimens may be helpful in diagnosis and future prospective studies of this tiny fascinating neoplasm. Recurrence was reported in $20 \%$ of cases ${ }^{[6]}$. There was also no recurrence after complete surgical excision of digital tumors. Complications following operative management included local infection and recurrence. However, excision of the surrounding capsule is necessary to prevent this local recurrence. Excisional treatment is difficult in glomangiomas; however, other treatment options include laser therapy, injection with hypertonic saline and sclerotherapy.

The only treatment for a glomus tumor is surgical excision. Recurrence is infrequent and is usually due to incomplete excision. Cutaneous and extra cutaneous glomus tumors are typically benign, and metastasis or malignant changes are extremely rare ${ }^{[1,2,4,8]}$. There was also no recurrence after complete surgical excision. Compared to the well-known subungual glomus tumor, extra digital glomus tumors may have atypical features, and the absence of objective findings often leads to a delay in diagnosis. A careful and comprehensive approach, including a thorough physical examination, comprehensive medical history, surveillance of the disease progress, and imaging studies, is essential upon clinical suspicion. Although glomus tumors of the forearm are rare, the neurologist and neurosurgeon should be aware of glomus tumors in the differential diagnosis of lesions presenting as peripheral neuropathic pain. This tiny mass can cause severe peripheral neuropathic pain that can be felt as electric waves radiating upwards or down wards along the limb and observed as nocturnal paroxysms provoked by cold. Non painful stimuli may produce more severe pain of longer duration (allodynia) and occasionally hyperalgesia, hyperesthesia and hyperpathia. A long history may unfortunately result in an improper diagnosis such as neuralgia, and psychosomatic pain. Clinical suspicion plays an important role in management of this lesion, and only early surgical excision alleviates the patient's pain.

\section{Future research}

The results presented in this study provide a wealth of information. This study used recent data from multiple surveys, to provide a detailed description of multiple demographic factors relating to the present topic. The descriptive results presented here raise a variety of issues that are ripe for further investigations. Our study has revealed suggestive relationships between these demographic factors; however, we were unable to examine the interrelationships among these factors as we are dealing with a rare case report. Future multivariate analyses, using statistical cohorts of digital and extra digital cases will allow us to come to a more nuanced understanding of these relationships. In addition, future research will explore what can be learned from using more appropriate definitions and new exploratory techniques. 


\section{References}

[1] Ruben RJ. History of glomus tumors: non chromaffin chemodectoma: a glimpse of biomedical Camelot. Acta Otolaryngol. 2007; 127(4): 411-416. http://dx.doi.org/10.1080/00016480601002088

[2] Gombos Z, Zhang PJ. Glomus tumor. Archives of pathology \& laboratory medicine. 2008; 132(9): 1448-52.

[3] Hazani R, Houle JM, Kasdan ML, et al. Glomus tumors of the hand. Eplasty. 2008; 8: e48.

[4] Tomak Y, Akcay I, Dabak N, et al. Subungual glomus tumors of the hand: diagnosis and treatment of 14 cases. Scand J Plast Reconstr Hand Surg. 2003; 37: 121-4. http://dx.doi.org/10.1080/02844310310005676

[5] Koç O, Kivrak AS, Paksoy Y. Subungual glomus tumor: magnetic resonance imaging findings. Australasian radiology. 2007; 51(Supplement s1): B107-B109. http://dx.doi.org/10.1111/j.1440-1673.2007.01797.x

[6] Koman LA, Rush DS, Smith BP, et al. Vascular disorders. In: Green DP, Hotchkiss RN, Peterson WC, Wolfe SW, editors. Green's Operative Hand Surgery. Philadelphia, PA: Elsevier. 2005: 2312.

[7] Kaylie DM, O’Malley M, Aulino JM, et al. Neurotologic surgery for glomus tumors. Otolaryngol. Clin. North Am. 2007; 40(3): 625-649. http://dx.doi.org/10.1016/j.otc.2007.03.009

[8] Weiss SW, Goldblum JR. Enzinger and Weiss's Soft Tissue Tumors, Mosby, Philadelpia, Pa, USA, 5th edition. 2008.

[9] Lee SK, Song DG, Choy WS. Intravascular glomus tumor of the forearm causing chronic pain and focal tenderness. Case Reports in Orthopedics. 2014; Article ID 619490, 4 pages. http://dx.doi.org/10.1155/2014/619490

[10] Lee S, Le H, Munk P, et al. Glomus tumor in the forearm: a case report and review of MRI findings. Journal Belge de Radiologie. 2010; 93(6): 292-295. http://dx.doi.org/10.5334/jbr-btr.342

[11] Nigam JS, Misra V, Singh A, et al. A glomus tumor arising from the flexor aspect of the forearm: a case report with review of the literature. Journal of Clinical and Diagnostic Research. 2012; 6(9): 1559-1561. PMid:23285458

[12] George SM, Morrison IK, Arrant PB, et al. Intravenous glomus tumor of the upper arm. BMJ Case Rep. 2012. http://dx.doi.org/10.1136/bcr.11.2011.5152

[13] Jiga LP, Rata A, Ignatiadis I, et al. Atypical venous glomangioma causing chronic compression of the radial sensory nerve in the forearm. A case report and review of the literature. Microsurgery. 2012; 32(3): 231-234. PMid:22407591 http://dx.doi.org/10.1002/micr.20983

[14] Rodríguez JM, Idoate MA, Pardo-Mind'an FJ. The role of mast cells in glomus tumors: report of a case of an intramuscular gloms tumor with a prominent mastocytic component. Histopathology. 2003; 42(3): 307-308. http://dx.doi.org/10.1046/j.1365-2559.2003.15355.x

[15] Gautam VK, Agarwal PK, Maini L, et al. Intraosseous glomus tumor in acromion process of scapula. Orthopedics. 2008; 31(4): 406.

[16] Giele H. Hildreth's test is a reliable clinical sign for the diagnosis of glomus tumor. J Hand Surg. 2002; 27(2): 157-8. PMid:12027491 http://dx.doi.org/10.1054/jhsb.2001.0724

[17] Lee DW, Yang JH, Chang S, et al. Clinical and pathological characteristics of extra digital and digital glomus tumors: a retrospective comparative study. J Eur Acad Dermatol Venereol. 2011; 25(12): 1392-7.

[18] Santos L, Chow C, Kennerson A. Glomus coccygeum may mimic glomus tumor. Pathology. 2002; 34(4): 339-43. PMid:12190292 http://dx.doi.org/10.1080/003130202760120508

[19] Schiefer TK, Parker WL, Anakwenze OA, et al. Extra digital glomus tumors: 20 years' experience. Mayo Clin Proc. 2006; 81(10): 1337-44. PMid:17036559 http://dx.doi.org/10.4065/81.10.1337

[20] Torrance N, Smith BH, Bennett MI, et al. The epidemiology of chronic pain of predominantly neuropathic origin. Results from a general population survey. J Pain. 2006; 7(4): 281-9. http://dx.doi.org/10.1016/j.jpain.2005.11.008

[21] Bouhassira D, Lantéri-Minet M, Attal N, et al. Prevalence of chronic pain with neuropathic characteristics in the general population. Pain. 2008; 136(3): 380-7. http://dx.doi.org/10.1016/j.pain.2007.08.013

[22] Hoekzema R, Zonneveld IM, Van der Wal AC. Type 2 segmental glomangiomas. Dermatology Online Journal. $2010 ; 16(1)$ : 8. PMid:20137750

[23] Chatterjee JS, Youssef AHK, Brown RM, et al. Congenital nodular multiple glomangioma: a case report. J Clin Pathol. 2006; 59(12): 1337.

[24] Brauer JA, Robert AR, Tzu J, et al. Glomuvenous Malformations (Familial generalized multiple glomangiomas. Dermatology Online Journal. 2011; 17(10): 9. PMid:22031635

[25] Bhaskaranand K, Navadgi BC. Glomus tumor of the hand. J Hand Surg. 2002; 27: 229-31. PMid:12074607 http://dx.doi.org/10.1054/jhsb.2001.0746

[26] Ham WK, Yun IS, Tark KC. Arch Plast Surg. 2013; 40(4): 392-396. http://dx.doi.org/10.5999/aps.2013.40.4.392 
[27] Folpe AL, Fanburg-Smith JC, Mietinen M, et al. Atypical and malignant glomus tumors: analysis of 52 cases, with a proposal for the reclassification of glomus tumors. Am J Surg Pathol. 2001; 25: 1-12. PMid:11145243 http://dx.doi.org/10.1097/00000478-200101000-00001

[28] Pérez de la FT, Vega C, Gutierrez PA, et al. Glomangiosarcoma of the hypothenar eminence: a case report. Chir Main. 2005; 24: 199-202. PMid:16121631 http://dx.doi.org/10.1016/j.main.2005.06.006

[29] Lamba G, Rafiyath SM, Kaur H, et al. Malignant glomus tumor of kidney: the first reported case and review of literature. Hum Pathol. 2011; 42(8): 1200-3. PMid:21333326 http://dx.doi.org/10.1016/j.humpath.2010.11.009 